(с) М.Н. Пешков ${ }^{1 *}$, Г.П. Пешкова², И.В. Решетов ${ }^{3}$

1Академия последипломного образования ФГБУ ФНКЦ ФМБА России, Москва

${ }^{2}$ Рязанский государственный медицинский университет имени академика И.П. Павлова, Рязань

ЗПервый Московский государственный медицинский университет им. И.М. Сеченова (Сеченовский Университет),

Москва

Сахарный диабет 2 типа (СД2) и рак предстательной железы являются широко распространенными заболеваниями во всем мире. СД2 выражается нарушением контроля гликемии, гиперинсулинемией, инсулинорезистентностью (ИР). Накопление глюкозы и липидов приводит к уменьшению плотности инсулиновых рецепторов и развитию ИР В жировой ткани. Это способствует развитию гиперинсулинемии, что подавляет распад жиров и приводит к прогрессированию ожирения. Развивается порочный круг: ИР $\rightarrow$ гиперинсулинемия $\rightarrow$ ожирение $\rightarrow$ ИР.

В исследованиях последнего времени показана сильная прямая корреляционная связь между уровнем инсулина натощак и смертностью от рака у мужчин. Это может быть особенно актуально у пациентов старше 65 лет, которые в первую очередь более склонны к развитию рака простаты, чем молодые пациенты. Стоит отметить, что именно инсулин, а не глюкоза, связан с риском развития онкологических заболеваний. Инсулин оказывает влияние на прогрессирование клеточного цикла, пролиферацию и метастатическую активность опухоли.

Гиперинсулинемия, которая часто возникает в результате андрогенной депривационной терапии (АДТ) - стандартного лечения рака предстательной железы, связана с высокой агрессивностью опухоли и более быстрой неэффективностью проводимой терапии - развитием кастрат-рефрактерного рака предстательной железы. Разумно предположить, что гиперинсулинемия - какими бы обстоятельствами она ни вызывалась, будь то АДТ или неправильное питание и другие факторы образа жизни - может оказывать такое же негативное влияние на передачу сигналов клетками.

Метаболический синдром - по существу, хронически повышенный уровень инсулина — тесно связан с рецидивом онкологических заболеваний и худшими исходами после лечения, что побудило исследователей усомниться в общепринятых диетических рекомендациях для больных раком, особенно когда они проходят лечение или выздоравливают после лечения, которое может включать рекомендации употреблять все, что поможет поддерживать или восстанавливать вес тела, независимо от содержания углеводов или его влияния на уровень инсулина.

Большое количество пациентов живут с гиперинсулинемией, но нормогликемией. Хроническая гиперинсулинемия является основной движущей силой кардиометаболических заболеваний, даже когда уровень глюкозы в крови в пределах референсных значений. Масштаб данной проблемы не осознан медицинским и научным сообществом.

КЛЮЧЕВЫЕ СЛОВА: рак предстательной железы; метаболический синдром; сахарный диабет; инсулинорезистентность; индекс массы тела; ожирение; дислипидемия

\title{
RELATIONSHIP BETWEEN PROSTATE CANCER AND TYPE 2 DIABETES MELLITUS
}

\author{
(c) Maxim N. Peshkov*, Galina P. Peshkova², Igor V. Reshetov ${ }^{3}$
}

${ }^{1}$ Academy of postgraduate education under FSCC of FMBA of Russia, Moscow, Russia

${ }^{2}$ Ryazan State Medical University, Ryazan, Russia

3.M. Sechenov First Moscow State Medical Univesity (Sechenov University), Moscow, Russia

Type 2 diabetes mellitus (DM2) and prostate cancer are widespread diseases throughout the world. Type II diabetes mellitus is accompanied by a deterioration in glycemic control, hyperinsulinemia, and insulin resistance (IR). The accumulation of glucose and lipids leads to a decrease in the density of insulin receptors and the development of insulin resistance in adipose tissue. This contributes to the development of hyperinsulinemia, which suppresses the breakdown of fat and leads to the progression of obesity. A vicious circle develops: insulin resistance $\rightarrow$ hyperinsulinemia $\rightarrow$ obesity $\rightarrow$ insulin resistance. Insulin influences the progression of the cell cycle, proliferation, and metastatic activity of the tumor.

Recent studies have shown a strong direct correlation between fasting insulin levels and cancer mortality in men. This may be especially true in patients over 65 , who are, in the first place, more likely to develop prostate cancer than younger patients. It should be noted that it is insulin, and not glucose, that is associated with the claim for the development of cancer. Hyperinsulinemia, which often occurs as a result of androgen deprivation therapy (ADT), the standard treatment for prostate cancer, is associated with a high tumor aggressiveness and faster treatment failure - the development of castrate-refractory prostate cancer. It is reasonable to assume that hyperinsulinemia - under whatever circumstances it is caused, whether due to ADT or due to inadequate nutrition and other lifestyle factors - can have the same negative effect on cellular signaling. 
Metabolic syndrome - essentially chronically elevated insulin levels - is closely associated with recurrence of cancer and worse post-treatment outcomes, which has led researchers to question generally accepted dietary guidelines for cancer patients, especially when they are undergoing treatment or recover from treatment, which may include recommendations to consume anything that will help maintain or restore body weight, regardless of sugar or carbohydrate content or its effect on insulin levels.

A large number of patients live with hyperinsulinemia, but normoglycemia. Chronic hyperinsulinemia is the main driver of cardiometabolic disease, even when blood sugar levels are within reference values. The scale of this problem is not recognized by the medical and scientific community.

KEYWORDS: prostate cancer; metabolic syndrome; diabetes mellitus; insulin resistance; Body Mass Index (BMI); obesity; dyslipidemia

Рак предстательной железы (РПЖ) является вторым по частоте диагностируемым злокачественным новообразованием в промышленно развитых странах и шестой по значимости причиной смерти от рака среди мужчин во всем мире (рак кожи занимает первое место по заболеваемости, в то время как рак легких является основной причиной смерти от рака) [1]. В России РПЖ является вторым наиболее часто диагностируемым раком и пятой по значимости причиной смерти от рака у мужчин [2]. Уровень заболеваемости РПЖ в западных странах в 10-15 раз выше, чем в азиатских, и большинство смертей, связанных с РПЖ, происходит в развитых странах; однако в последние несколько десятилетий заболеваемость РПЖ и связанная с ним смертность в Юго-Восточной Азии, по-видимому, возросли, поскольку этот регион постепенно начал охватывать западный образ жизни, включая сидячие привычки и диету с высоким содержанием жиров [3].

Патогенез. Хотя в патогенезе РПЖ выделяют несколько причин, установленными факторами риска являются возраст, раса и семейный анамнез [4]. Данные экспериментальных трансляционных исследований подтверждают гипотезу о том, что нарушение метаболизма глюкозы играют значительную роль в развитии и прогрессировании новообразований [5]. Несколько метаболических нарушений, включающих ожирение [6] и гиперинсулинемию [7], связаны с риском развития рака простаты. Такие факторы образа жизни, как избыточное потребление энергии и отсутствие физической активности, обычно связанные с РПЖ, способствуют ухудшению метаболического профиля, что приводит к развитию резистентности к инсулину, висцеральному ожирению, провоспалению и гормональным изменениям [8]. Метаболический профиль создает внешнюю среду, способствующую более агрессивному росту опухоли простаты. Данные факторы образа жизни являются модифицируемыми, дают возможность изменить внешнюю опухолевую среду и обеспечить эффективный метод снижения риска развития РПЖ.

Пациенты с раком простаты, как правило, подвергаются повышенному риску развития метаболических нарушений, таких как метаболический синдром (MC), сердечно-сосудистые заболевания [9] и сахарный диабет [9], данные риски часто связывают с лечением. В незлокачественных популяциях метаболические последствия, включая резистентность к инсулину, висцеральное ожирение, низкую мышечную массу и провоспаление, связанные с агрессивным развитием РПЖ, также связаны с развитием вторичных болезненных состояний, таких как сердечно-сосудистые заболевания и диабет, что предполагает циклическая модель метаболического заболевания и РПЖ (рис. 1). Тем не менее, большая часть литературы не в состоянии интегрировать многие факторы, такие как перечисленные выше, которые способствуют метаболическому здоровью у пациентов с раком простаты на протяжении всей траектории лечения (риск выживания), что приводит к значительным пробелам в литературе, исследующей роль метаболического здоровья при РПЖ.

В данном обзоре обобщена литература по метаболизму глюкозы и инсулинорезистентности (ИР); описаны эти процессы у пациентов с раком простаты на протяжении всего развития заболевания; выявлены отсутствующие элементы в литературе и выработаны новые гипотезы о роли метаболизма глюкозы и инсулина, ИР при раке простаты.

Метаболический синдром. МС является распространенным клиническим состоянием со сложной этиологией, включая потребление большого количества жира, малоподвижный образ жизни и генетические факторы. Кроме того, МС представляет собой группу факторов риска сердечно-сосудистых и метаболических осложнений, которые включают висцеральное ожирение, гипертонию, гипергликемию, низкий уровень холестерина липопротеинов высокой плотности (холестерин ЛПВП) и гипертриглицеридемию [10]. МС был предложен в качестве одной из основных причин изменчивости географической заболеваемости и смертности от рака простаты. В частности, связь между МС и РПЖ была постулирована на основе роста числа случаев РПЖ среди азиатских мигрантов, что позволяет предположить, что вестернизация является важным фактором риска для РПЖ [11]. В связи с этим метаболические нарушения, характерные для западных районов, такие как диабет и ожирение, могут играть важную роль в развитии и прогрессировании РПЖ.

Ведущими механизмами, связывающими МС и РПЖ, могут быть изменение инсулина и инсулиноподобного фактора роста I (IGF-I), модификации путей половых стероидов, такие как повышение уровня эстрадиола в сыворотке, концентрация связывающего половые гормоны глобулина и снижение уровня свободного тестостерона [12]. Кроме того, хроническое воспаление предстательной железы, наблюдаемое у пациентов с МС, связано со средой, обогащенной цитокинами, медиаторами воспаления и факторами роста, что может привести к неконтролируемому пролиферативному ответу. Фактически повышенный уровень циркулирующих цитокинов, связанных с МС, а также изменения лептина и адипонектина были предварительно связаны с канцерогенезом РПЖ [8].

Текущие доклинические и клинические исследования МС и РПЖ по-прежнему противоречивы и не смогли определить реальное влияние $\mathrm{MC}$ и/или его отдельного 


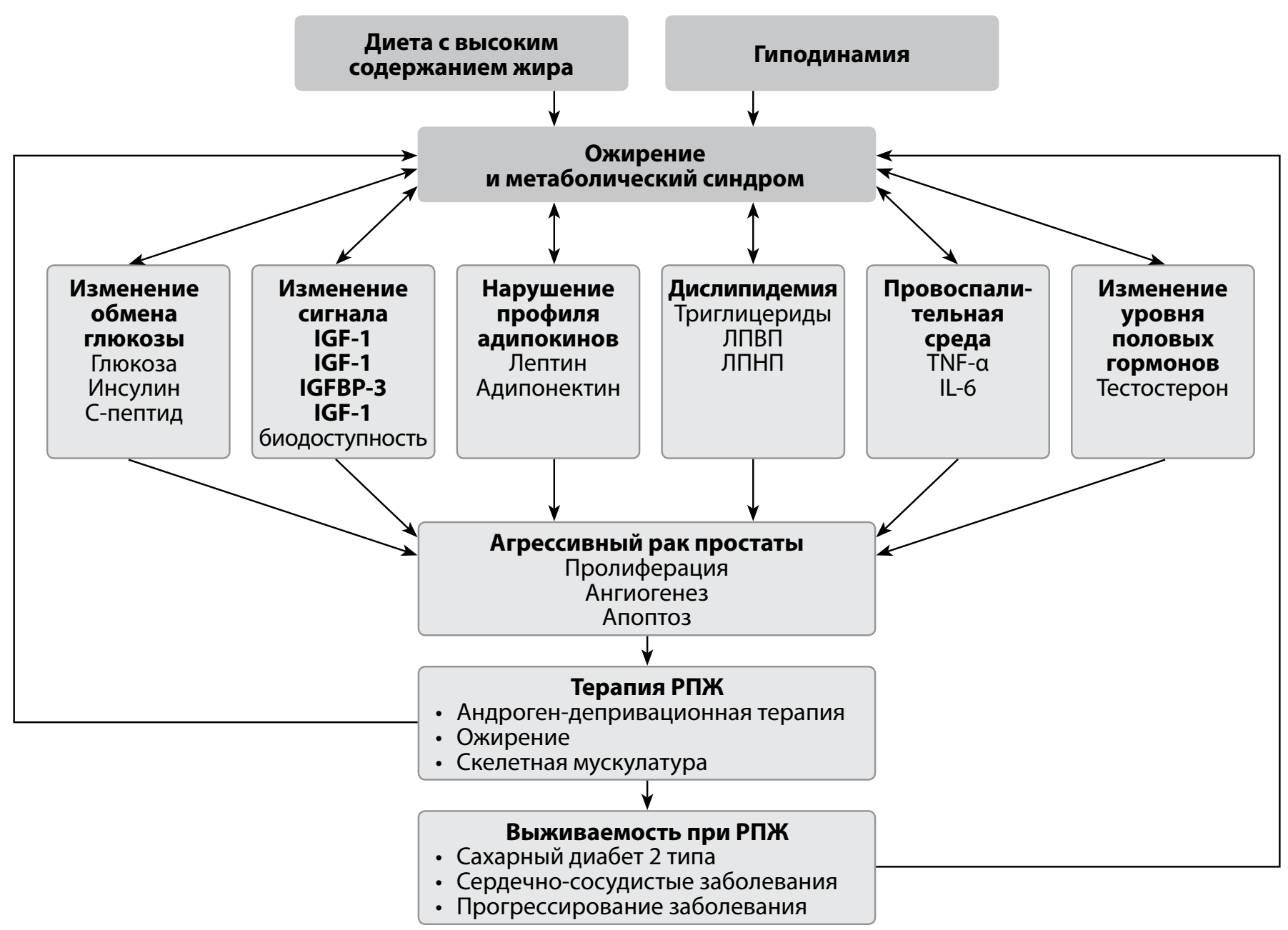

Рисунок 1. Циклическая, интегрированная модель рака простаты.

Примечания: ЛПВП - липопротеины высокой плотности, лПнП — липопротеины низкой плотности, РПж — рак предстательной железы

компонента на частоту возникновения и агрессивность РПЖ. Цели этого систематического обзора и метаанализа состоят в том, чтобы оценить влияние МС на частоту возникновения и риск развития РПЖ и проанализировать их роль в развитии агрессивных свойств рака простаты.

Сахарный диабет 2 типа (СД2). СД2 повышает риск смерти при наличии нескольких солидных злокачественных новообразований, включая колоректальный рак и рак молочной железы, но в случае рака простаты существуют противоречивые данные. Влияние ранее существовавшего СД2 также изучалось в отношении степени и стадии РПЖ при представлении с противоречивыми результатами. Более того, появляются свидетельства того, что наличие СД2 и других метаболических нарушений (дислипидемия, гипертония, ожирение) связано с более быстрым прогрессированием РПЖ. Эта взаимосвязь еще более осложняется тем фактом, что стандартное лечение прогрессирующего РПЖ, андрогенная депривационная терапия (АДТ), приводит к увеличению заболеваемости СД2 [13], а также к развитию СД2 у лиц с ранее существовавшим предиабетом.

\section{РОЛЬ САХАРНОГО ДИАБЕТА 2 ТИПА В РАЗВИТИИ РАКА ПРЕДСТАТЕЛЬНОЙ ЖЕЛЕЗЫ}

Хроническая гипергликемия при СД2 сопровождается повреждением, дисфункцией и недостаточностью различных органов, особенно глаз, почек, нервов, сердца и кровеносных сосудов [14].

В настоящее время хорошо известно, что при СД2 увеличивается риск развития злокачественных новообразований [15]. Некоторые исследования показывают, что люди с СД2 в два раза чаще умирают от рака, чем люди без него. Однако при РПЖ наблюдается обратная отрицательная корреляционная связь, данные опубликованы в нескольких метаанализах [15].

S. Bonovas и соавт. опубликовали метаанализ, изучающий СД2 и риск развития РПЖ (2004 г.). Они включили 14 исследований и пришли к выводу, что СД2 дает статистически значимое снижение относительного риска (ОР) развития РПЖ на 9\% [16]. В 2006 г. опубликованы данные метаанализа, проведенного J.S. Kasper и соавт., которые включили результаты 19 исследований. Авторы сообщили об обратной зависимости аналогичной величины с ОР 0,84 (95\% ДИ 0,76-0,93) [17]. После этого D. Bansal и соавт. опубликовали обновленный метаанализ, включающий 45 исследований с участием 8,1 млн пациентов и включением 132331 случаев РПЖ, в которых также сообщается об обратной связи с ОР 0,86 (95\% ДИ 0,80-0,92) [18]. Недавно P. Gang и соавт. опубликовали обновленный метаанализ с обзором литературы до апреля 2012 г. включительно. Этот метаанализ содержал 56 исследований, в которых сообщалось об обратной связи, OP 0,88 (95\% ДИ 0,82-0,93) [19]. 

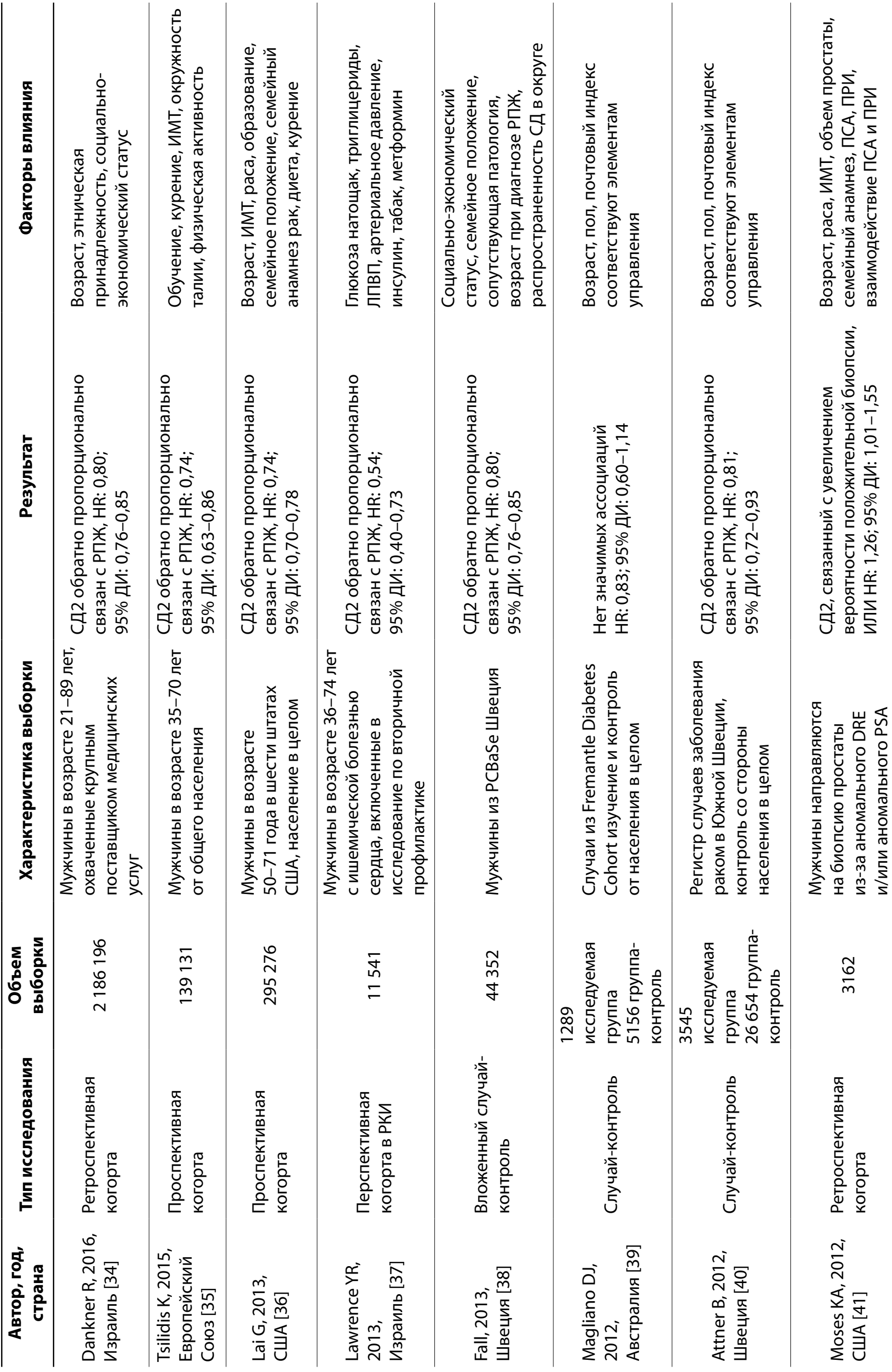

ì
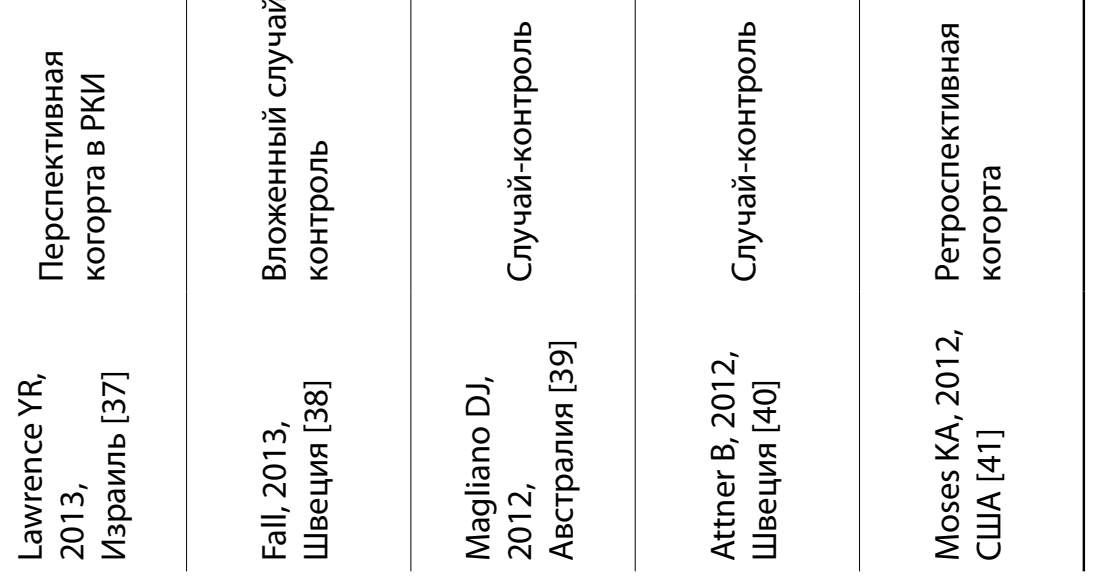
Из приведенных 8 исследований (табл. 1) пять были когортными [20-23, 27], три исследования «случай-контроль» [24-26]. Три исследования были выполнены на пациентах европейской популяции, два из США, два из Израиля и одно из Австралии.

Объединенные исследования включают 2716302 случаев наблюдения. В 6 исследованиях сообщили об обратной корреляционной связи между заболеванием СД2 и риском развития РПЖ [20-24, 26], в 1 об отсутствии ассоциации [25], и еще в 1 сообщалось о положительной ассоциации [27]. В 6 исследованиях, в которых сообщалось об обратной связи, показаны схожие показатели ассоциации по величине 20\% снижения риска развития РПЖ у лиц с манифестирующим СД2 по сравнению с теми, у кого не было данного заболевания. Одна статья, в которой не сообщается о статистически значимой связи, представляет собой исследование типа «случай-контроль», в котором случаи СД2 представлены пациентами, включенными в исследование The Fremantle Diabetes Study (FDS) [25]. Это наименьшее из исследований, с 1289 случаями наблюдения и 5156 пациентами контрольной группы, что может объяснить статистическую незначимость их результатов (ОР 0,83, 95\% ДИ: 0,60-1,14) — хотя направление и величина ассоциации соответствуют другим исследованиям. Исследование, в котором сообщалось о положительной связи, представляет собой ретроспективный обзор 3162 мужчин, которым была сделана биопсия простаты из-за повышенного уровня простатического специфического антигена (ПСА) и/или аномального результата пальцевого ректального исследования (ПРИ) [27]. Этот дизайн отличается от других исследований, описанных ранее, которые основаны на общей, а не на выбранной популяции, с результатами биопсии простаты. Эта неоднородность в дизайне может объяснять наличие $26 \%$ увеличения шансов положительной биопсии у пациентов с СД2 по сравнению с пациентами без СД (ОШ 1,26; 95\% ДИ: 1,01-1,55).

\section{РОЛЬ ИНСУЛИНА В РАЗВИТИИ РАКА ПРЕДСТАТЕЛЬНОЙ ЖЕЛЕЗЫ}

Гиперинсулинемия связана со многими видами рака, включая рак простаты. ИР в периферической ткани приводит к гиперинсулинемии для поддержания нормального уровня глюкозы в крови [28]. Однако инсулин также является мощным фактором роста и связан с ускоренным ростом опухоли простаты и большим распространением заболевания [29]. Показано, что опухоль простаты высокой группы риска характеризуется большим количеством инсулиновых рецепторов (IR) на своей мембране, а также повышенной экспрессией изоформы IR-A как в моделях in vitro, так и in vivo [30]. Активация IR стимулирует как путь PI3K/Akt/mTOR, так и путь MAP/ERK-киназы, что в конечном итоге приводит к пролиферации и миграции клеток и ингибированию апоптоза [29]. Таким образом, повышенные концентрации инсулина в циркулирующей крови, такие как связанные с ИР, в сочетании с повышенным числом рецепторов инсулина на агрессивных опухолях предстательной железы подтверждают гипотезу о значении инсулина в развитии и росте рака простаты.
Широкий спектр исследований продемонстрировал положительную связь между гиперинсулинемией, риском развития РПЖ и ее большей агрессивностью. Хотя не все исследования подтверждают эту взаимосвязь, концентрации инсулина натощак, близкие к верхним диапазонам референсных значений, были положительно связаны с раком простаты [31]. С другой стороны, отрицательная связь обычно наблюдается с риском развития РПЖ у пациентов с длительным анамнезом СД2 и снижением концентрации инсулина из-за гибели $\beta$-клеток [32]. Метформин, обычно используемый для контроля уровня глюкозы в крови у пациентов с СД2 путем снижения выработки глюкозы в печени и повышения чувствительности периферических тканей к поглощению глюкозы, также связан со сниженным риском рака простаты [33]. Несмотря на эти данные, подтверждающие роль инсулина в развитии РПЖ, метаанализ не выявил существенных связей между лечением инсулином и риском развития рака простаты [34].

Другие факторы, участвующие в метаболизме глюкозы, также вовлечены в развитие РПЖ. IGF-I и IGF-II, как было показано, увеличивают риск РПЖ и действуют по тем же сигнальным путям, что и инсулин. Хотя они могут связываться с IR, у них есть собственный IGF-IR, который может активировать сигнальные каскады. Показано, что IGF-I и IGF-II высвобождаются из опухолевых клеток и вызывают аутокринную и паракринную функцию в дополнение к их эндокринной функции.

Протеин-3, связывающий инсулиноподобный фактор роста (IGFBP-3), - это пептид, участвующий в регуляции роста клеток. Более 95\% имеющегося в сыворотке крови IGF-1 связано с белками-носителями, которые подразделяются на 6 классов. Самый важный из них - белокноситель IGFBP-3. Данный пептид обладает равной аффинностью как к IGF-1, так и к IGF-2. Увеличение количества инсулиноподобного белка-3 обратно пропорционально риску развития рака простаты [35].

Повышенные концентрации С-пептида натощак связаны с риском развития РПЖ и более высокой степенью рака [36]. О секреции инсулина судят по содержанию С-пептида, когда в бета-клетке из проинсулина отделяется инсулин. Поскольку С-пептид и инсулин секретируются в эквимолярных концентрациях, оценить уровень секреции инсулина можно по уровню С-пептида. Измерения С-пептида позволяют проводить различие между повышенной секрецией инсулина или сниженным клиренсом, выявляя потенциальный механизм гиперинсулинемии.

P.F. Zamboni и соавт. [37] продемонстрировали, что концентрации инсулина натощак и гомеостатическая модель оценки ИР (HOMA-IR) были выше у пациентов с РПЖ с различной степенью тяжести заболевания по сравнению с неонкологическими пациентами из группы контроля со сходным индексом массы тела (ИМТ), несмотря на нормальный уровень концентрации глюкозы через 2 часа при проведении перорального глюкозотолерантного теста (ПГТТ). Поскольку уровень инсулина имеет значение при агрессивном росте РПЖ, здесь важно определить, повышены ли уровни инсулина и С-пептида для управления концентрациями глюкозы во время ПГТT. K.M. Di Sebastiano и coавт. [38] продемонстрировали, что у пациентов с РПЖ при постановке диагноза концентрация глюкозы через 2 часа ПГТТ была более низкой и имела более высокий уровень инсулина и С-пептида по сравнению с мужчинами того же 
возраста и массы тела без рака. U.Y. Tekdoğan и соавт. [39] обнаружили, что IGF-1 и IGFBP-3 не различались у пациентов с РПЖ и доброкачественной гиперплазией предстательной железы (ДГПЖ) во время ПГТТ; однако в группе ДГПЖ концентрация инсулина была значительно выше, чем у больных раком [39]. Эти данные показывают, что инсулин может способствовать росту клеток простаты, но его роль в развитии малигнизации неясна.

\section{МЕХАНИЗМЫ ВЗАИМОСВЯЗИ САХАРНОГО ДИАБЕТА 2 ТИПА И РАКА ПРЕДСТАТЕЛЬНОЙ ЖЕЛЕЗЫ}

Биологический механизм, лежащий в основе обратной связи между СД2 и риском развития РПЖ, до конца не изучен.

Во-первых, у людей с СД2 происходят метаболические изменения, которые могут защитить от РПЖ. Теория канцерогенеза предполагает, что длительная гиперинсулинемия приводит к снижению инсулинсвязывающих белков и, следовательно, к увеличению свободного IGF-1, что приводит к клеточным изменениям, которые могут привести к канцерогенезу через усиление митоза и снижение апоптоза. Имеются как лабораторные, так и эпидемиологические данные, подтверждающие прямую корреляционную связь: повышение уровня инсулина приводит к увеличению риска развития РПЖ. У пациентов с СД2 первоначально отмечается повышенный уровень инсулина, со временем развивается гипоинсулинемия. Следовательно, пациенты с СД2, которые имеют более низкие уровни инсулина, с течением времени будут защищены с точки зрения риска развития РПж [40]. В других исследованиях сообщалось об усилении обратной связи между рисками СД2 и РПЖ с продолжительностью СД2 [41], что подтверждает данную гипотезу.

Во-вторых, длительная гипоинсулинемия также приводит к снижению уровня лептина, гормона, участвующего в энергетическом гомеостазе, высокая концентрация которого связана с риском развития РПЖ [42]. Тем не менее, нет опубликованных исследований данной взаимосвязи между уровнями инсулина и лептина при СД2 и риском развития РПЖ.

\section{ИЗМЕНЕНИЕ МЕТАБОЛИЗМА ГЛЮКОЗЫ ПРИ ЛЕЧЕНИИ РАКА ПРЕДСТАТЕЛЬНОЙ ЖЕЛЕЗЫ, ВЛИЯНИЕ НА ВЫЖИВАЕМОСТЬ}

АДТ является распространенным методом лечения РПЖ высокого риска и связана с развитием СД2 [43]. Сывороточные концентрации инсулина натощак и концентрация глюкозы натощак повышаются уже в течение 12 нед и продолжают повышаться после 12 мес АДТ. M.R. Smith и соавт. обнаружили, что концентрация инсулина значительно повышена во время ПГТ на 12-й неделе АДТ по сравнению с исходным уровнем, несмотря на то, что ответы на глюкозу оставались такими же, предполагая снижение чувствительности к инсулину после 12 нед АДТ. Снижение индекса чувствительности к инсулину и увеличение уровня гликированного гемоглобина, который является маркером долговременных нарушений метаболизма глюкозы, также наблюдается у пациентов с РПЖ после 12 нед АДТ. Эпидемиологические данные свидетельствуют о 61\% увеличении риска развития диабета после АДТ, пик которого наступает через 3 года после лечения [44].
Механизмы, лежащие в основе взаимосвязи АДТ и развития резистентности к инсулину, плохо охарактеризованы. Из-за комплексного характера ИР и состава тела может быть трудно различить эти признаки АДТ независимо друг от друга. Тем не менее, основная гипотеза предполагает, что взаимодействие между специфическими адипокинами (цитокинами, которые секретируются накопленным дисфункциональным жиром) и низкий уровень тестостерона влияют на развитие резистентности к инсулину. Провоспалительные цитокины, полученные из жировой ткани, включая интерлейкин-6 (IL-6) и фактор некроза опухоли-а (TNF-a), ассоциированы с СД2 [45]. Было показано, что низкие концентрации тестостерона способствуют резистентности к инсулину у незлокачественных гипогонадальных мужчин с метаболическим синдромом. Напротив, введение тестостерона может снизить концентрацию инсулина в плазме и HOMA-IR. И соответственно снизить уровни IL-6 и TNF-a [46]. Возможно, что перекрестные помехи между половыми стероидами и провоспалительными цитокинами во время АДТ могут влиять на развитие резистентности к инсулину у пациентов. Однако неизвестно, являются ли повышенные уровни провоспалительных цитокинов причиной резистентности к инсулину или являются следствием АДТ и измененных уровней тестостерона.

Существуют также доказательства того, что другие виды лечения могут увеличить риск диабета у выживших после РПЖ. M.S.Y.Thong и соавт. [47] исследовали частоту диабета в первые 5 лет после постановки диагноза РПЖ и продемонстрировали, что СД2 был выявлен у 50,2\% пациентов, перенесших радикальную простатэктомию, 21,4\% получали лучевую терапию, 16,3\% проходили активное наблюдение и только 12,1\% получали гормональную терапию в качестве основного вида лечения [47]. Эти данные свидетельствуют о том, что все пациенты с РПЖ, а не только те, кто получает АДТ, подвержены риску развития диабета в условиях выживания. Однако, что примечательно, подавляющее большинство исследований было сосредоточено на метаболических эффектах гормональной терапии.

\section{ПОТЕНЦИАЛЬНАЯ РОЛЬ ОЖИРЕНИЯ И СОСТАВА ТЕЛА В РЕЗИСТЕНТНОСТИ К ИНСУЛИНУ У ПАЦИЕНТОВ С РАКОМ ПРОСТАТЫ}

Ожирение вовлечено в развитие нарушенного метаболизма глюкозы, резистентности к инсулину и в конечном итоге СД2. Многочисленные метаанализы сообщают о небольшом, но значительном положительном увеличении относительного риска развития РПЖ в отношении ожирения (обычно оцениваемого по ИМТ) [48-50]. Существуют дополнительные данные, свидетельствующие о наличии конкретной связи между ожирением и агрессивным РПЖ [50]. Литература, исследующая связь между РПЖ низкого риска или локализованным и ожирением, остается неубедительной. A. Discacciati и соавт. [51] сообщили о защитном эффекте ожирения от локализованного рака простаты (ОР 0,94; 95\% ДИ 0,91-0,97), но о повышенном риске агрессивного рака простаты при ожирении (ОР 1,09; 95\% ДИ 1,02-1,16). Ожирение связано с повышенным риском биохимического рецидива (повышение уровня ПСА в крови после хирургического лечения или лучевой терапии) и специфической смертностью от рака 
простаты [48]. Увеличение ИМТ на 5 кг/м² было связано с увеличением биохимического рецидива на 17-21\% [49] после радикальной простатэктомии и лучевой терапии под действием внешнего луча и с 15\% увеличением канцерспецифической смертности от РПЖ после радикальной простатэктомии [48].

Предполагается, что инсулин и IGF-1 объясняют связь между РПЖ и ожирением. Повышенные концентрации инсулина и свободного IGF-1, которые связаны с ожирением [52], приводят к увеличению пролиферации опухоли, снижению апоптоза опухоли и переходу опухолевых клеток предстательной железы в состояние, устойчивое к андрогенам. Ускоренный рост опухоли на моделях ксенотрансплантата РПж был зарегистрирован в результате вызванной диетой гиперинсулинемии. Тучные мужчины имеют более низкий уровень тестостерона, что также может объяснить связь между ожирением и агрессивным РПЖ. Низкий уровень тестостерона создает среду, способствующую росту более агрессивных опухолей, не зависящих от тестостерона [53].

Адипокины, лептин и адипонектин также могут опосредовать связь между РПЖ и ожирением и участвовать в развитии резистентности к инсулину. Уровень лептина повышен при ожирении, и было показано, что он увеличивает пролиферацию клеток РПЖ и ингибирует апоптоз [42] как в чувствительных к андрогенам (LNCaP), так и в устойчивых к андрогенам (PC-3 и DU145) клеточных линиях $[42,54]$. В противоположность лептину адипонектин обладает противоопухолевым действием, а концентрации адипонектина в сыворотке снижаются у лиц с ожирением [55]. Пациенты с раком простаты, демонстрирующие более низкие концентрации адипонектина в сыворотке [56], продемонстрировали обратную зависимость между гистологической степенью и стадией заболевания и уровнями адипонектина в плазме [56]. Предполагается, что защитный эффект адипонектина происходит посредством передачи сигналов AMPK, NF-кB, PPAR-a и MAPK, а также нижестоящих модераторов JNK и STAT3 [57]. Взятые вместе, эти данные свидетельствуют о важной роли адипокинов и риска развития агрессивного рака простаты, однако необходимы дальнейшие исследования, чтобы выяснить влияние этих сложных взаимодействий на резистентность к инсулину.

Следует отметить, что ИМТ, который обычно используется для оценки роли ожирения при РПЖ, является грубым показателем ожирения. Точные инструменты, такие как компьютерная томография (KT), ультразвук, магнитно-резонансная томография (МРТ) или двухэнергетическая рентгеновская абсорбциометрия, должны использоваться для четкой характеристики мышечной массы и ожирения при раке простаты [58]. Существует возможность оценки изображений, полученных во время диагностики и последующего наблюдения, так как запрашивать КТ и МРТ для оценки состава тела не всегда возможно.

На скелетные мышцы приходится >75\% выведения глюкозы [53]. Большая часть литературы, исследующей скелетные мышцы при РПЖ, изучает взаимосвязь между АДТ и потерей скелетных мышц. Андрогены являются основным анаболическим стимулом в скелетных мышцах у мужчин, и, следовательно, АДТ вызывает значительную потерю мышц и увеличение жировой ткани [59]. В неопухолевых популяциях эти характеристики состава тела связаны с развитием резистентности к инсулину и метаболического синдрома. Гипогонадизм является независимым предиктором гиперинсулинемии и МС [60]. Литература демонстрирует четкую связь между использованием АДТ и потерей скелетных мышц [59], однако в нашем понимании характеристик скелетных мышц существует значительный разрыв при диагностике и изменениях во время лечения в метаболизме мышц, которые могут привести к нарушениям метаболизма глюкозы.

\section{ЗАКЛЮЧЕНИЕ}

Недостаток инсулина и ИР играют центральную роль в развитии СД2. В начале данного обзора рассматривается влияние ИР и существующего СД2 на частоту возникновения РПЖ, что подтверждает ранее опубликованные результаты, свидетельствующие о том, что СД2 может оказывать защитное воздействие на риск возникновения РПЖ. Приведено несколько потенциальных биологических механизмов и возможных предубеждений, объясняющих эту обратную связь.

Исследовано влияние существующего СД2 на тип опухолевого процесса. В некоторых исследованиях высказано предположение, что обратная связь наблюдается только при раке с низким риском и что у пациентов с СД2 на самом деле более высок риск развития РПЖ. Однако это не согласуется с существующим метаанализом, и в настоящее время нельзя сделать вывод о влиянии СД2 на риск развития РПЖ у разных классов и стадий. Существующая литература свидетельствует, что СД2 постоянно ассоциируется с повышенным риском смертности от всех причин и от РПЖ. Отношения между СД2 и РПЖ дополнительно осложняются взаимодействием между двумя состояниями и их лечением. Исследована взаимосвязь между АДТ и СД2, и между всеми исследованиями существует хорошее соответствие, причем все они демонстрируют повышение риска развития СД2.

Эпидемиологические данные, изучающие взаимосвязь между воздействием метформина и РПЖ, однако, менее убедительны. Это не показывает снижения ни окончательного риска РПЖ или улучшения РПЖ, ни смертности от всех причин, связанных с метформином.

В финальной части обзора рассмотрен вопрос, изучающий влияние РПЖ и его терапии на течение существующего СД2. Необходимо продолжить исследование в данной области.

\section{ДОПОЛНИТЕЛЬНАЯ ИНФОРМАЦИЯ}

Финансирование работы. Обзорно-аналитическая работа по подготовке рукописи проведена на личные средства автора.

Конфликт интересов. Обзор является частью диссертационного исследования М.Н. Пешкова. Остальные авторы декларируют отсутствие явных и потенциальных конфликтов интересов, связанных с публикацией настоящей статьи.

Участие авторов. Пешков М.Н. - сбор, анализ данных литературы, написание статьи; Пешкова Г.П., Решетов И.В. - редактирование статьи. Все авторы прочли и одобрили финальную версию рукописи перед публикацией. 


\section{СПИСОК ЛИТЕРАТУРЫ | REFERENCES}

1. Siegel RL, Miller KD, Jemal A. Cancer statistics, 2019. CA Cancer J Clin. 2019;69(1):7-34. doi: https://doi.org/10.3322/caac.21551.

2. Состояние онкологической помощи населению России в 2019 году. / Под ред. Каприна А.Д., Старинского В.В., Шахзадовой А.О. - М.: МНИОИ им. П.А. Герцена - филиал ФГБУ «НМИЦ радиологии» Минздрава России; 2020. 236 с. [State of oncological care in Russia in 2020. Ed by Kaprin AD, Starinskiy W, Shachzadova AO. Moscow: MNIOI im. P.A. Gertsena — filial FGBU "NMIRTS radiologii" Minzdrava Rossii; 2020. 236 p. (In Russ.)].

3. Hsing AW, Sakoda LC, Chua S Jr. Obesity, metabolic syndrome, and prostate cancer. Am J Clin Nutr. 2007;86:s843-s857. doi: https://doi.org/10.1093/ajcn/86.3.843S

4. Esposito K, Chiodini P, Capuano A, et al. Effect of metabolic syndrome and its components on prostate cancer risk: meta-analysis. J Endocrinol Invest. 2013;36:132-139. doi: https://doi.org/10.1007/BF03346748

5. Esposito K, Chiodini P, Colao A, et al. Metabolic syndrome and risk of cancer: a systematic review and meta-analysis. Diabetes Care. 2012;35:2402-2411. doi: https://doi.org/10.2337/dc12-0336

6. Keum N, Greenwood DC, Lee DH, et al. Adult Weight Gain and Adiposity-Related Cancers: A Dose-Response Meta-Analysis of Prospective Observational Studies. JNCI J Nat/ Cancer Inst. 2015;107(2). doi: https://doi.org/10.1093/jnci/djv088

7. Pandeya DR, Mittal A, Sathian B, et al. Role of hyperinsulinemia in increased risk of prostate cancer: a case control study of the Kathmandu Valley. Asian Pac J Cancer Prev. 2014;15:1031-1033. doi: https://doi.org/10.7314/apjcp.2014.15.2.1031

8. De Nunzio C, Aronson W, Freedland SJ, et al. The correlation between metabolic syndrome and prostatic diseases. Eur Urol. 2012;61:560-570. doi: https://doi.org/10.1016/j.eururo.2011.11.013

9. Nguyen PL, Alibhai SMH, Basaria S, et al. Adverse effects of androgen deprivation therapy and strategies to mitigate them. Eur Urol. 2015; 67:825-836. doi: https://doi.org/10.1016/j.eururo.2014.07.010

10. Alberti KG, Eckel RH, Grundy SM, et al. Harmonizing the metabolic syndrome: a joint interim statement of the International Diabetes Federation Task Force on Epidemiology and Prevention; National Heart, Lung, and Blood Institute; American Heart Association; World Heart Federation; International Atherosclerosis Society; and International Association for the Study of Obesity. Circulation. 2009;120:1640-1645. doi: https://doi.org/10.1161/CIRCULATIONAHA.109.192644

11. Hsing AW, Devesa SS. Trends and patterns of prostate cancer: what do they suggest? Epidemiol Rev. 2001;23:3-13. doi: https://doi.org/10.1093/oxfordjournals.epirev.a000792

12. Han JH, Choi NY, Bang SH, et al. Relationship between serum prostate-specific antigen levels and components of metabolic syndrome in healthy men. Urology. 2008;72:749-754. doi: https://doi.org/10.1016/j.urology.2008.01.084

13. Keating NL, O'Malley AJ, Smith MR. Diabetes and cardiovascular disease during androgen deprivation therapy for prostate cancer. J Clin Oncol. 2006;24(27):4448-4456. doi: https://doi.org/10.1200/JCO.2006.06.2497

14. Дедов И.И., Шестакова М.В., Майоров А.Ю., и др. Алгоритмы специализированной медицинской помощи больным сахарным диабетом: Клинические рекомендации (Вып. 9) // Сахарный диабет. 2019. — T. 22. — №S1. — C.1-144. [Dedov II, Shestakova MV, Mayorov AYu, et al. Standards of specialized diabetes care. Diabetes Mellitus. 2019;22(S1):1144 (In Russ.)]. doi: https://doi.org/10.14341/DM221S1

15. Tsilidis KK, Kasimis JC, Lopez DS, et al. Type 2 diabetes and cancer: umbrella review of meta-analyses of observational studies. BMJ. 2015;350:g7607. doi: https://doi.org/10.1136/bmj.g7607

16. Bonovas S, Filioussi K, Tsantes A. Diabetes mellitus and risk of prostate cancer: a meta-analysis. Diabetologia. 2004;47(6):1071-1078. doi: https://doi.org/10.1007/s00125-004-1415-6

17. Kasper JS, Giovannucci E. A meta-analysis of diabetes mellitus and the risk of prostate cancer. Cancer Epidemiol Biomarkers Prev. 2006;15(11):2056-2062. doi: https://doi.org/10.1158/1055-9965.EPI-06-0410

18. Bansal D, Bhansali A, Kapil G, et al. Type 2 diabetes and risk of prostate cancer: a meta-analysis of observationa studies. Prostate Cancer Prostatic Dis. 2013;16(2):151-158. doi: https://doi.org/10.1038/pcan.2012.40

19. Jian Gang P, Mo L, Lu Y, et al. Diabetes mellitus and the risk of prostate cancer: an update and cumulative meta-analysis. Endocr Res. 2015;40(1):54-61. doi: https://doi.org/10.3109/07435800.2014.934961

20. Dankner R, Boffetta P, Balicer RD, et al. Time-dependent Risk of cancer after a diabetes diagnosis in a cohort of 2.3 million adults. Am J Epidemiol. 2016;183(12):1098-1106. doi: https://doi.org/10.1093/aje/kwv290

21. Tsilidis KK, Allen NE, Appleby PN, et al Diabetes mellitus and risk of prostate cancer in the European prospective investigation into cancer and nutrition Int J Cancer. 2015;136(2):372-381. doi: https://doi.org/10.1002/ijc.28989

22. Lai GY, Park Y, Hartge P, et al. The association between selfreported diabetes and cancer incidence in the NIH-AARP diet and health study. J Clin Endocrinol Metab. 2013;98(3):E497-E502. doi: https://doi.org/10.1210/jc.2012-3335

23. Lawrence YR, Morag O, Benderly M, et al. Association between metabolic syndrome, diabetes mellitus and prostate cancer risk. Prostate Cancer Prostatic Dis. 2013;16(2):181-186. doi: https://doi.org/10.1038/pcan.2012.54

24. Fall K, Garmo H, Gudbjornsdottir S, et al. Diabetes mellitus and prostate cancer risk; a nation wide case-control study within PCBaSe Sweden. Cancer Epidemiol Biomarkers Prev. 2013;22(6):1102-1109 doi: https://doi.org/10.1158/1055-9965.EPI-12-1046

25. Magliano DJ, Davis WA, Shaw JE, et al. Incidence and predictors of all-cause and site-specific cancer in type 2 diabetes: the Fremantle diabetes study. Eur J Endocrinol. 2012;167(4):589-599. doi: https://doi.org/10.1530/EJE-12-0053

26. Attner B, Landin-OlssonM, LithmanT, et al. Cancer among patients with diabetes, obesity and abnormal blood lipids: a population-based register study in Sweden. Cancer Causes Control. 2012;23(5):769-777 doi: https://doi.org/10.1007/s10552-012-9946-5

27. Moses K A, Utuama O A, Goodman M, et al. The association of diabetes and positive prostate biopsy in a US veteran population Prostate Cancer. Prostatic Dis. 2012;15(1):70-74 doi: https://doi.org/10.1038/pcan.2011.40

28. Samueal VT, Shulman GI. The pathogenesis of insulin resistance: integrating signaling pathways and substrate flux. J Clin Invest 2016;1(26):12-22. doi: https://doi.org/10.1172/JCI77812

29. Arcidiacono B, liritano S, Nocera A, et al. Insulin Resistance and Cancer Risk: An Overview of the Pathogenetic Mechanisms. Exp Diabetes Res. 2012;2012:1-12. doi: https://doi.org/10.1155/2012/789174

30. Perks CM, Zielinska HA, Wang J, et al. Insulin receptor isoform variations in prostate cancer cells. Front Endocrinol. 2016;7:132. doi: https://doi.org/10.3389/fendo.2016.00132

31. Albanes D, Weinstein SJ, Wright ME, et al. Serum insulin, glucose, indices of insulin resistance, and risk of prostate cancer. J Nat/ Cancer Inst. 2009;101:1272-1279. doi: https://doi.org/10.1093/jnci/djp260

32. Darbinian JA, Ferrara AM, Van Den Eeden SK, et al. Glycemic status and risk of prostate cancer. Cancer Epidemiol Biomarkers Prev. 2008;17:628-635. doi: https://doi.org/10.1158/1055-9965.EPI-07-2610

33. Preston MA, Riis AH, Ehrenstein V, et al. Metformin use and prostate cancer risk. Eur Urol. 2014;66:1012-1020. doi: https://doi.org/10.1016/j.eururo.2014.04.027

34. Chen Y, Chen Q, Wang Z, Zhou J. Insulin Therapy and Risk of Prostate Cancer: a Systematic Review and Meta-Analysis of Observational Studies. PLoS One. 2013;8(11):e81594. doi: https://doi.org/10.1371/journal.pone.0081594

35. Cattabiani C, Basaria S, Ceda GP. Luci, et al. Relationship between testosterone deficiency and cardiovascular risk and mortality in adult men. J Endocrinol Invest. 2012;35:104-120. doi: https://doi.org/10.3275/8061

36. Di Sebastiano KM, Pinthus JH, Duivenvoorden WCM, et al. Elevated c-peptides, abdominal obesity and abnormal adipokine profile are associated with higher Gleason scores in prostate cancer. Prostate. 2017;77:211-221. doi: https://doi.org/10.1002/pros.23262

37. Zamboni PF, Simone M, Passaro A, et al. Metabolic profile in patients with benign prostate hyperplasia or prostate cancer and normal glucose tolerance. Horm Metab Res. 2003;35:296-300. doi: https://doi.org/10.1055/s-2003-41305

38. Di Sebastiano KM, Bell KE, Mitchell AS, et al. Glucose metabolism during the acute prostate cancer treatment trajectory: The influence of age and obesity. Clin Nutr. 2018;37(1):195-203. doi: https://doi.org/10.1016/j.clnu.2016.11.024

39. Tekdoğan UY, Bağcioğlu M, Özcan S, et al. The effect of oral glucose tolerance test on insulin and some related indicators in elderly male patients with prostate cancer and benign prostate hyperplasia. Turkish J Geriatrics. 2015;18:10-14. 
40. Hammarsten J, Hogstedt B. Hyperinsulinaemia: a prospective risk factor for lethal clinical prostate cancer. Eur J Cancer. 2005;41(18):2887-2895. doi: https://doi.org/10.1016/j.ejca.2005.09.003

41. Rodriguez C, Patel AV, Mondul AM, et al. Diabetes and risk of prostate cancer in a prospective cohort of US men. Am J Epidemiol. 2005;161(2):147-152. doi: https://doi.org/10.1093/aje/kwh334

42. Noda T, Kikugawa T, Tanji N, et al. Long-term exposure to leptin enhances the growth of prostate cancer cells. Int J Oncol. 2015;46(4):1535-1542. doi: https://doi.org/10.3892/ijo.2015.2845

43. Keating NL, O'Malley AJ, Freedland SJ, et al. Diabetes and cardiovascular disease during androgen deprivation therapy: observational study of veterans with prostate cancer. J Nat/ Cancer Inst. 2010;102:39-46. doi: https://doi.org/10.1093/jnci/djp404

44. Crawley D, Garma H, Rudman S, et al. Association between duration and type of androgen deprivation therapy and risk of diabetes in men with prostate cancer. Int J Cancer. 2016;139:2698-2704. doi: https://doi.org/10.1002/ijc.30403

45. Dennis RJ, Maldonado D, Rojas MX, et al. Inadequate glucose control in type 2 diabetes is associated with impaired lung function and systemic inflammation: a cross-sectional study. BMC Pulm Med. 2010;10:38. doi: https://doi.org/10.1186/1471-2466-10-38

46. Kalinchenko SY, Tishova YA, Mskhalaya GJ, et al. Effects of testosterone supplementation on markers of the metabolic syndrome and inflammation in hypogonadal men with the metabolic syndrome: the double-blinded placebo-controlled Moscow study. Clin Endocrinol (Oxf) 2010;73:602-612. doi: https://doi.org/10.1111/j.1365-2265.2010.03845.x

47. Thong MSY, van de Poll-Franse L, Hoffman RM et al. Diabetes mellitus and health-related quality of life in prostate cancer: 5-year results from the Prostate Cancer Outcomes Study. BJU Int. 2010;107:1223-1231. doi: https://doi.org/10.1111/j.1464-410X.2010.09861.X

48. Renehan AG, Tyson M, Egger M, et al. Body-mass index and incidence of cancer: a systematic review and meta-analysis of prospective observational studies. Lancet. 2008;371:569-578. doi: https://doi.org/10.1093/annonc/mdr603

49. Cao Y, Ma J. Body mass index, prostate cancer-specific mortality, and biochemical recurrence: a systematic review and meta-analysis. Cancer Prev Res. 2011;4:486-501. doi: https://doi.org/10.1158/1940-6207.CAPR-10-0229
50. Allott EH, Masko EM, Freedland SJ. Obesity and prostate cancer: weighing the evidence. Eur Urol. 2013;63:800-809. doi: https://doi.org/10.1016/j.eururo.2012.11.013

51. Discacciati A, Orsini N, Wolk A. Body mass index and incidence of localized and advanced prostate cancer - a dose-response meta-analysis of prospective studies. Ann Oncol. 2012;23:1665-1671. doi: https://doi.org/10.1093/annonc/mdr603

52. Rowlands MA, Gunnell D, Harris R, et al. Circulating insulin-like growth factor peptides and prostate cancer risk: a systematic review and meta-analysis. Int J Cancer. 2009;124:2416-2429. doi: https://doi.org/10.1002/ijc.24202

53. Kapoor D, Malkin CJ, Channert KS, et al. Androgens insulin resistance and vascular disease in men. Clin Endocrinol (Oxf). 2005;63:239-250. doi: https://doi.org/10.1111/j.1365-2265.2005.02299.x

54. Alshaker $\mathrm{H}$, Sacco K, Alfraidi A, et al. Leptin signalling, obesity and prostate cancer: molecular and clinical perspective on the old dilemma. Oncotarget. 2015;6:35556-35563. doi: https://doi.org/10.18632/oncotarget.5574

55. Nigro E, Scudiero O, Monaco ML, et al. New Insight into Adiponectin Role in Obesity and Obesity-Related Diseases. Biomed Res Int. 2014;2014:1-14. doi: https://doi.org/10.1155/2014/658913

56. Goktas S, Yilmaz MI, Caglar K, et al. Prostate cancer and adiponectin. Urologia. 2005;65:1 168-1172. doi: https://doi.org/10.3233/CBM-160467

57. Kelesidis I, Kelesidis T, Mantzoros CS. Adiponectin and cancer: a systematic review. Br J Cancer. 2006;94:1221-1225. doi: https://doi.org/10.1038/sj.bjc.6603051

58. Paris M, Mourtzakis M. Assessment of skeletal muscle mass in critically ill patients: considerations fot the utility of computed tomography images and ultrasonography. Curr Opin Clin Nutr Metab Care. 2016;19:125-130. doi: https://doi.org/10.1097/MC0.0000000000000259

59. Chen Z, Maricic M, Nguyen P, et al. Low bone density and high percentage of body fat among men who were treated with androgen deprivation therapy for prostate carcinoma. Cancer. 2002;95:2136-2144. doi: https://doi.org/10.1002/cncr.10967

60. Muller M, Grobbee DE, Tonkelaar I, et al. Endogenous sex hormones and metabolic syndrome in aging men. J Clin Endocrinol Metab. 2005;90:2618-2623. doi: https://doi.org/10.1210/jc.2004-1158.

\section{ИНФОРМАЦИЯ ОБ АВТОРАХ [AUTHORS INFO]}

*Пешков Максим Николаевич, к.м.н. [Maxim N. Peshkov, MD, PhD]; адрес: Россия, 125371, Москва, Волоколамское ш., д. 91 [address: 91 Volokolamskoye Highway street, 125371 Moscow, Russia]; ORCID: https://orcid.org/0000-0002-1497-8646; eLibrary SPIN: 1691-1478; e-mail: drpeshkov@gmail.com

Пешкова Галина Петровна, К.M.H. [Galina P. Peshkova, MD, PhD]; ORCID: https://orcid.org/0000-0002-3336-4025; eLibrary SPIN: 6849-5407; e-mail: gppeshkova@gmail.com

Решетов Игорь Владимирович, д.м.н. [lgor V. Reshetov, MD, PhD]; ORCID: https://orcid.org/0000-0002-5739-3170; eLibrary SPIN: 3845-6604; e-mail: reshetoviv@mail.ru

\section{ЦИТИРОВАТЬ:}

Пешков М.Н., Пешкова Г.П., Решетов И.В. Взаимосвязь рака предстательной железы и сахарного диабета второго типа // Сахарный диабет. — 2021. — T. 24. — №6. — C. xxx-xxx. doi: https://doi.org/10.14341/DM12672

\section{TO CITE THIS ARTICLE:}

Peshkov MN, Peshkova GP, Reshetov IV. Relationship between prostate cancer and type 2 diabetes mellitus. Diabetes Mellitus. 2021;24(6):xxx-xxx. doi: https://doi.org/10.14341/DM12672 